\title{
Teoría de los cuidados de Kristen Swanson: revisión de literatura
}

\author{
Kristen Swanson's theory of care: literature review
}

\section{Uma teoria do cuidado de Kristen Swanson: revisão da literatura}

\author{
Isabel Beristain-García ${ }^{1 *}$ \\ https://orcid.org/0000-0001-9112-9421 \\ Alicia Álvarez-Aguirre 2 \\ (D) https://orcid.org/0000-0001-5538-7634 \\ Mayra Itzel Huerta-Baltazar ${ }^{3}$ \\ (Dhttps://orcid.org/0000-0003-0908-424X \\ Leticia Casique-Casique ${ }^{4}$ \\ (iDhttps://orcid.org/0000-0002-0532-4819
}

1. Estudiante de Doctorado en Ciencias de Enfermería. División de Ciencias de la Salud e Ingenierías. Universidad de Guanajuato. Campus Celaya Salvatierra. Guanajuato, México.

2. Profesora del Departamento de Enfermería Clínica. Universidad de Guanajuato. División de Ciencias de la Salud e Ingenierías. Campus Celaya Salvatierra. Guanajuato, México.

3. Profesora Investigadora. Universidad Michoacana de San Nicolás de Hidalgo. Facultad de Enfermería. Michoacán, México.

4. Profesora Investigadora. Universidad de Guanajuato. División de Ciencias de la Salud e Ingenierías, Campus Celaya Salvatierra. Departamento de Enfermería y Obstetricia. Guanajuato, México.

*Autor para correspondencia: beristaingarcia@yahoo.com.mx

Recibido: 03/06/2020

Aceptado: 14/12/2021

\section{Resumen}

Introducción: La disciplina de enfermería ha buscado a lo largo de los años fundamentar las intervenciones de su quehacer aplicando teorías propias de la disciplina, como la teoría de los cuidados, la cual proporciona la pauta trasladarla a las áreas de acción. Objetivo: Analizar la evidencia científica disponible sobre la aplicación de la teoría de los cuidados de Kristen Swanson en el quehacer de enfermería. Metodología: Revisión de la literatura que aborda la teoría de los cuidados, se utilizó la metodología de Cooper, se 
consultaron diferentes bases de datos: IMBIOMED, CUIDEN, ScienceDirect, Fundación Index, MEDLINE, ciberINDEX, SciELO, Redalyc, entre otras. Los descriptores empleados fueron: enfermería, atención de enfermería, teoría de enfermería, en idioma español, inglés y portugués entre los años 2015 y 2019, se incluyó todo tipo de literatura, obteniendo una muestra de 42 artículos y disertaciones. Resultados: La mayoría de los documentos analizados no plasman por completo los procesos básicos de cuidado que plantea Swanson. La teoría ha demostrado ser útil en el área asistencial, elaboración de programas de intervención con neonatos, pacientes pos-operados de cardiopediatría, lactancia materna, cuidados en el puerperio y modificación de la actitud hacia la donación de órganos. Conclusión: La teoría se considera esencial para la valoración integral, adoptar sus fundamentos y conceptos es de gran utilidad, ya que favorece la generación de un primer espacio de aprendizaje, resulta útil para brindar un acompañamiento al paciente y su familia, y ofrecerles estrategias de mejora. Se requiere realizar más investigaciones de intervención, cualitativas y de docencia.

Palabras clave: Enfermería; atención de enfermería; Teoría de enfermería (DeCS).

\begin{abstract}
Introduction: Over the years, the nursing discipline has sought to base the interventions of its work by applying theories that are specific to this discipline, such as the theory of care, which provides the guideline to transfer it to the areas of action. Objective: Analyze the scientific evidence available on the application of Kristen Swanson's theory of care in nursing practice. Methodology: The Cooper's methodology was used in order to review literature regarding the theory of care; different databases were consulted: IMBIOMED, CUIDEN, ScienceDirect, Fundación Index, MEDLINE, ciberINDEX, SciELO, Redalyc, among others. The descriptors used were: nursing, nursing care, nursing theory, in Spanish, English and Portuguese between the years 2015 and 2019; all types of literature were included, obtaining a sample of 42 papers and dissertations. Results: Most of the documents assessed do not fully capture the basic processes of care proposed by Swanson. The theory has proven to be useful in the area of care, development of intervention programs with neonates, post-operative cardiopediatric patients, breastfeeding, puerperium care and modification of attitudes towards organ donation. Conclusion: The theory is considered essential for integral assessment, and adopting its foundations and concepts is of great utility, since it favors the generation of a first learning space; moreover, it is useful to provide accompaniment to the patient and his family, and to offer them strategies for improvement. More intervention, qualitative and teaching research is required.
\end{abstract}

Key words: Nursing; Nursing theory; Nursing care (DeCS).

\begin{abstract}
Abstrato
Introdução: A disciplina de enfermagem tem procurado ao longo dos anos basear as intervenções de seu trabalho aplicando teorias específicas à disciplina, como a teoria do cuidado, que fornece a diretriz para transferi-la para as áreas de ação. Objetivo: Analisar as evidências científicas disponíveis sobre a aplicação da teoria de Kristen Swanson sobre o cuidado na prática da enfermagem. Metodologia: Revisão da literatura sobre a teoria dos cuidados, foi utilizada a metodologia de Cooper, foram consultadas diferentes bases de dados: IMBIOMED, CUIDEN, ScienceDirect, Fundación Index, MEDLINE, ciberINDEX, SciELO,
\end{abstract}


Redalyc, entre outros. Os descritores utilizados foram: enfermagem, cuidados de enfermagem, teoria da enfermagem, em espanhol, inglês e português entre os anos de 2015 e 2019, todos os tipos de literatura foram incluídos, obtendo-se uma amostra de 42 artigos e dissertações. Resultados: A maioria dos documentos analisados não capta completamente os processos básicos de cuidados propostos pela Swanson. A teoria provou ser útil na área de cuidados, desenvolvimento de programas de intervenção com recém-nascidos, pacientes cardiopediátricos pós-operatórios, amamentação, cuidados com o puerpério e modificação de atitudes em relação à doação de órgãos. Conclusão: A teoria é considerada essencial para a avaliação integral, a adoção de seus fundamentos e conceitos é de grande utilidade, pois favorece a geração de um primeiro espaço de aprendizado, é útil para proporcionar acompanhamento ao paciente e sua família, e oferecer-lhes estratégias de melhoria. É necessária mais intervenção, pesquisa qualitativa e pedagógica.

Palavras-chave: Enfermagem; Cuidados de enfermagem; Teoria de enfermagem (DeCS).

\section{Introducción}

La disciplina de enfermería ha ido evolucionando desde Florencia Nightingale hasta la época actual, y en esa búsqueda de identidad en la década de los cincuenta del siglo $X X$, enfermería inicia la separación del conocimiento médico con el conocimiento propio de la disciplina; este inicio llevó a la necesidad de desarrollar teorías propias de enfermería ${ }^{(1)}$, existe literatura general y de enfermería que brinda diferentes conceptos de teoría (2); la palabra "teoría" proviene del griego theoreo que significa "mirar", "observar" (3).

Las teorías permiten la generación del conocimiento, y de esta manera avanzar en la conformación de un cuerpo propio de conocimientos disciplinares, estás han permitido a enfermería mejorar su capacidad profesional y disciplinar, a través del conocimiento y los métodos aplicados de forma sistemática para mayor probabilidad de éxito ${ }^{(4)}$. Las teorías tienen como fin, describir, explicar, predecir y controlar o prescribir el fenómeno ${ }^{(5)}$.

La teoría es la segunda entidad teórica, en nivel de abstracción, según Fawcett ${ }^{(6)}$, y suele confundirse con modelo conceptual, porque se habla indiscriminadamente de ambos términos como si significaran lo mismo; al referirse a una teoría suelen nombrarla como modelo, sin embargo; el modelo conceptual es descriptivo y si se quiere hacer investigación con él debe fragmentarse en partes lógicas y que sus conceptos sean operativos; aunque muchas teóricas no hacen ninguna diferencia entre ambos ${ }^{(3)}$. Las teorías se diferencian en 
grandes teorías, teorías y teorías de rango medio. La teoría de mayor amplitud es la gran teoría, la de menor amplitud la microteoría, y se ubica dentro del concepto de teoría de rango medio acorde al termino en español TRM. Las TRM se ocupan de fenómenos específicos de enfermería, surgen y reflejan tanto la práctica como los procesos clínicos ${ }^{(7)}$.

Kristen Swanson en el año 1991, planteó la "Teoría de los cuidados (TC)" (1) considerada TRM o nivel medio; cabe mencionar que la teoría derivó empíricamente de una investigación fenomenológica. La teoría de Swanson resulta útil aplicando los 5 procesos básicos de cuidado que propone la teorista ${ }^{(8)}$; a) Conocer: lo importante es conocer a las personas con respeto, entendiendo sin prejuicios sus vidas, valorando cada actividad que lleven a la apropiación del conocimiento. b) Estar con: se inicia con la identificación de la persona, como única e individual, se acompaña y estimula la expresión de emociones y de percepciones acerca del momento que se estaba viviendo. c) Hacer por: estar emocionalmente presente con el otro y hacer por el otro lo que haríamos por nosotros mismos. Para esto se inicia un proceso de educación, acompañamiento, búsqueda de ayuda, según las necesidades individuales identificadas. d) Mantener las creencias: se descubre, a través de interacción, creencias, experiencias, destrezas, habilidades y conocimientos que son importantes para las personas y que pueden ser redescubiertos, orientados o situados, para garantizar prácticas de cuidado adecuadas en esta etapa de la vida. e) Posibilitar: se educa a las personas sobre recomendaciones específicas según la necesidad identificada; además se incluye en la atención, otros profesionales que acompañaron con su disciplina.

La TC es clara, sencilla y generalizable a toda relación enfermera-persona, en cualquier contexto clínico ${ }^{(1)}$; da la pauta para que enfermería la traslade a las áreas de acción donde se desempeña; enfermería a nivel asistencial (clínica o domiciliaria), administrativo, docencia e investigación ${ }^{(9)}$. Para proporcionar un cuidado humanizado se requiere que los profesionales de enfermería cuenten con experiencia, habilidad, destreza y capacidad en el conocimiento integral y holístico de la persona en el área de su desempeño; de este modo la TC resulta ser una herramienta útil ya que ha sido contrastada en investigación, formación y práctica clínica; 
su utilidad se ha demostrado en la investigación, educación y práctica clínica (1); pero actualmente ¿es así? Por este motivo surge el objetivo de analizar la evidencia disponible de la literatura científica sobre la aplicación de la TC de Kristen Swanson en las cuatro áreas del quehacer de enfermería, mediante la metodología de Cooper ${ }^{(10)}$.

\section{Metodología}

Se realizó una revisión de la literatura sobre las publicaciones científicas que abordaron la TC; considerando los siete pasos de Cooper: ${ }^{(10)}$ 1) Formulación del problema, para este caso la pregunta fue ¿cuál ha sido la aplicación de la TC en las áreas de administración, docencia, asistencial e investigación en enfermería?; 2) Búsqueda de la literatura, en lo conducente se procedió a indagar en diferentes bases de datos: Pubmed, IMBIOMED, EBSCO, Elsevier, CUIDEN, ScienceDirect, Hemeroteca Cantárida, Fundación Index, Biblioteca Virtual en salud, MEDLINE; ciberINDEX, SciELO, Redalyc, se consideraron estas bases por su calidad: entre 2015 y 2019; utilizando los descriptores DeCS en español: enfermería, atención de enfermería, teoría de enfermería, en inglés (nursing, nursing care, nursing theory), y portugués (enfermagem, cuidado de enfermagem, teoría de enfermagem); se utilizó el operador boleano AND. Se incluyeron artículos cuantitativos, cualitativos, ensayos clínicos, revisiones, meta-análisis o serie de casos, que utilizaron la teoría de Kristen Swanson, así como disertaciones de pregrado y posgrado; el único criterio para excluir artículos fue que hayan sido publicados antes del 2015.

3) Recabar información de los estudios para el propósito planteado, por lo que se obtuvieron los aspectos generales como título, año de publicación, autores y resumen; posterior se realizó lectura del texto completo para encontrar sesgos o duplicidad. 4) Evaluación de la calidad de los estudios, en consecuencia, se realizó una lectura crítica identificando la TC y que cumpliera con los apartados de introducción, resumen, metodología utilizada, resultados, conclusiones, sugerencias o recomendaciones y bibliografía. 5) Análisis e interpretación de los resultados de los estudios, de tal forma que se procedió a leer cada uno de los artículos identificando: autor, año, país, instrumento que utilizaron, muestra, edad de los participantes, diseño de 
investigación y en qué área de enfermería fue enfocada la teoría 6) Interpretación de la evidencia, en atención a lo cual se analizaron a profundidad aspectos metodológicos de los estudios: variables, definiciones conceptuales, tipo de estudio (empírico o teórico), tipo de metodología (cuantitativa, cualitativa o mixta) y diseño (descriptivo, correlacional, casos y controles etc.); objetivo, hipótesis o propósito del estudio, población de estudio, selección de los participantes (aleatoria o por conveniencia), instrumentos de medición empleados (confiables y válidos), procedimientos, limitaciones durante los procedimientos, congruencia entre los estadísticos y los objetivos o hipótesis planteados. 7) Presentación de resultados, se identificaron 62 artículos en las bases de datos consultadas, se excluyeron 20 artículos por estar fuera del rango de los años comprendidos, el total de artículos incluidos fue de 42 para el reporte final (Figura 1).

Figura 1. Revisión de literatura

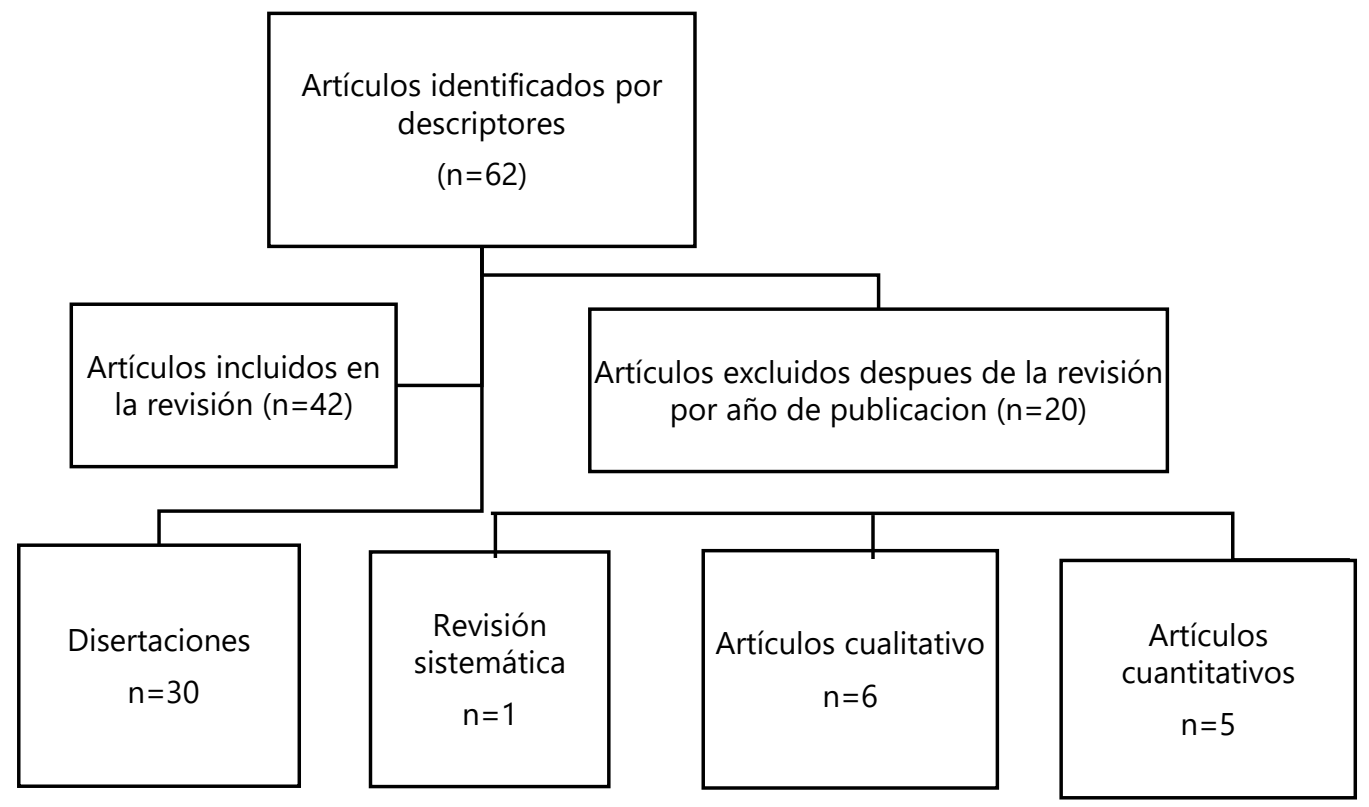

Fuente: Elaboración propia

\section{Resultados}

En relación con las características generales de los artículos, un 14.2\% correspondió a investigaciones cualitativas de artículos originales con enfoque: fenomenológico, ${ }^{(11)}$, uno sin especificar el método utilizado 
(12), teoría fundamentada ${ }^{(13)}$, reflexivo ${ }^{(14)}$, historia de vida ${ }^{(15)}$ y hermenéutico ${ }^{(16)}$, un $71.4 \%$ eran disertaciones, el $11.9 \%$ eran investigaciones cuantitativas y un $2.3 \%$ era revisión sistemática. Las características principales se muestran en la tabla 1 basada en el manual Cochrane ${ }^{(17)}$.

En cuanto a los procesos básicos de cuidado de la TC, ha demostrado que apoya en la modificación de la actitud hacia la donación de órganos, ya que al aplicar un post test presentó diferencia estadística significativa y su intervención fue elaborada con cada uno de los procesos básicos ${ }^{(24)}$. Así también, se ha propuesto un modelo de cuidado bajo el enfoque teórico de la TC para el adulto mayor sano a través de un instrumento validado para el adulto mayor sustentado en la TC (53). En el $38.0 \%$ del total de las investigaciones no visualizan el desarrollo de la TC en resultados y conclusiones a pesar de ser el sustento teórico; otras investigaciones mencionan la TC en el marco teórico o conceptual y en el resto del trabajo no se vuelve a mencionar. Se colocan los procesos básicos en el árbol de problemas y objetivos sin embargo al momento de referirse a la intervención no se visualizan ${ }^{(48)}$.

Tabla 1. Características de las disertaciones analizadas, $n=30$

\begin{tabular}{|c|c|c|c|c|}
\hline $\begin{array}{c}\text { Autores } \\
\text { /grado } \\
\text { académico }\end{array}$ & $\begin{array}{l}\text { Año, } \\
\text { país }\end{array}$ & Métodos y objetivo & Participantes & $\begin{array}{l}\text { Resultados } \\
\text { Principales }\end{array}$ \\
\hline $\begin{array}{l}\text { Aquino AY, et } \\
\text { al. }{ }^{24} \\
\text { Licenciatura }\end{array}$ & $\begin{array}{l}2018, \\
\text { Perú }\end{array}$ & $\begin{array}{l}\text { Cuantitativo, diseño cuasi } \\
\text { experimental con pre y post } \\
\text { prueba con grupo control. } \\
\text { Aplicar la TC para modificar la } \\
\text { actitud hacia la donación de } \\
\text { órganos en estudiantes de quinto } \\
\text { de secundaria de la I. E. Manuel } \\
\text { Benito Linares Arenas, Arequipa, } \\
2017 \text {. }\end{array}$ & $\begin{array}{l}\text { Grupo control=16 } \\
\text { estudiantes } \\
\text { Grupo experimental=15 } \\
\text { estudiantes. }\end{array}$ & $\begin{array}{l}\text { La actitud hacia la donación de } \\
\text { órganos en los estudiantes del grupo } \\
\text { control en el pre y post test, no } \\
\text { presentó diferencia estadística } \\
\text { significativa. } \\
\text { La actitud hacia la donación de } \\
\text { órganos del grupo experimental en } \\
\text { el pre y post test presentó diferencia } \\
\text { estadística significativa. }\end{array}$ \\
\hline $\begin{array}{l}\text { Mogollon } A L \text {, } \\
\text { et al. }{ }^{25} \\
\text { Licenciatura }\end{array}$ & $\begin{array}{l}\text { 2019, } \\
\text { Perú }\end{array}$ & $\begin{array}{l}\text { Cualitativo con abordaje } \\
\text { metodológico estudio de caso. } \\
\text { Analizar y comprender la } \\
\text { percepción del cuidado } \\
\text { enfermero en pacientes } \\
\text { hospitalizados en el servicio de } \\
\text { cirugía: según la TC, Chiclayo- } \\
\text { 2019. }\end{array}$ & $\begin{array}{l}12 \text { pacientes hospitalizados } \\
\text { del servicio de cirugía, entre } \\
18 \text { a } 60 \text { años, con estancia } \\
\text { hospitalaria de } 3 \text { días. }\end{array}$ & $\begin{array}{l}\text { Contrastes ante el llamado del } \\
\text { paciente; diferencias en el hacer } \\
\text { enfermero: comunicación y } \\
\text { administración de medicamentos, } \\
\text { generando alternativas para un } \\
\text { mejor cuidado del paciente. }\end{array}$ \\
\hline
\end{tabular}

Continúa.... 
Tabla 1. Continuación....

\begin{tabular}{|c|c|c|c|c|}
\hline $\begin{array}{l}\text { Romero } \mathrm{AR} \text {, } \\
\text { et al. } 26 \\
\text { Licenciatura }\end{array}$ & $\begin{array}{l}2019 \\
\text { Perú }\end{array}$ & $\begin{array}{l}\text { Descriptiva, correlacional, } \\
\text { corte transversal. Establecer la } \\
\text { relación entre el nivel de } \\
\text { conocimientos y prácticas } \\
\text { sobre lactancia materna con el } \\
\text { estado nutricional de lactantes } \\
\text { menores de seis meses en el } \\
\text { Centro de Salud de Liberación } \\
\text { Social. }\end{array}$ & $\begin{array}{l}92 \text { lactantes } \\
\text { menores y sus } \\
\text { respectivas } \\
\text { madres. }\end{array}$ & $\begin{array}{l}55.4 \% \text { de madres tuvieron alto nivel de } \\
\text { conocimientos y el } 44.6 \% \text { nivel de } \\
\text { conocimientos medio. }\end{array}$ \\
\hline $\begin{array}{l}\text { Ding CS, et } \\
\text { al. } 27 \\
\text { Licenciatura }\end{array}$ & $\begin{array}{l}2018 \\
\text { país } \\
\text { faltó }\end{array}$ & $\begin{array}{l}\text { Cualitativo, exploratorio, } \\
\text { descriptivo. } \\
\text { Describir cuales son las } \\
\text { practicas alimentarias de los } \\
\text { cuidadores con menores de } 5 \\
\text { años en la comunidad. }\end{array}$ & $\begin{array}{l}\text { Cuidadores con } \\
\text { menores de } 5 \\
\text { años. }\end{array}$ & $\begin{array}{l}\text { Resultaron tres categorías: } 1 \text { condición de } \\
\text { acceso a los alimentos, } 2 \text { en la población se } \\
\text { observa desconocimiento y } 3 \text { contexto cultural }\end{array}$ \\
\hline $\begin{array}{l}\text { Bajaña AZ, et } \\
\text { al. } 28 \\
\text { Licenciatura }\end{array}$ & $\begin{array}{l}\text { 2018, } \\
\text { Ecuador }\end{array}$ & $\begin{array}{l}\text { Estudio cualitativo } \\
\text { interpretativo socio-crítico. } \\
\text { Proponer las intervenciones de } \\
\text { enfermería en la seguridad del } \\
\text { neonato en alojamiento } \\
\text { conjunto del Hospital Pablo } \\
\text { Arturo Suarez. }\end{array}$ & $\begin{array}{l}9 \text { cuidadoras de } \\
\text { las cuales } 5 \\
\text { fueron madres de } \\
\text { familia y } 4 \text { madres } \\
\text { comunitarias. }\end{array}$ & $\begin{array}{l}\text { La seguridad del neonato en alojamiento } \\
\text { conjunto se ve afectada debido a factores } \\
\text { extrínsecos como la falta de asistencia de } \\
\text { calidad, evidenciado por el déficit de talento } \\
\text { humano y la inadecuada infraestructura del } \\
\text { servicio exponiendo al neonato a riesgos de } \\
\text { asfixia, caída, infección, y el riesgo de ictericia. }\end{array}$ \\
\hline $\begin{array}{l}\text { Chinguel YK, } \\
\text { et al. }{ }^{29} \\
\text { Licenciatura }\end{array}$ & $\begin{array}{l}\text { 2018, } \\
\text { Perú }\end{array}$ & $\begin{array}{l}\text { Cuasi experimental. } \\
\text { Determinar la efectividad del } \\
\text { cuidado domiciliario de } \\
\text { enfermería con enfoque } \\
\text { familiar en la adherencia del } \\
\text { paciente al tratamiento de } \\
\text { hipertensión arterial en el }\end{array}$ & 80 participantes. & $\begin{array}{l}\text { Al inicio del estudio mostraron un nivel de bajo } \\
\text { adherencia terapéutica en el grupo de } \\
\text { intervención con un } 62.5 \% \text { y el grupo control } \\
\text { con } 55 \%, 3 \text { meses después de la aplicación del } \\
\text { cuidado domiciliario el grupo de intervención } \\
\text { presentó una alta adherencia con } 52.5 \% \text { y en el } \\
\text { grupo control no hubo cambios significativos. }\end{array}$ \\
\hline
\end{tabular}
centro de salud materno infantil Dr. Enrique Martin Altuna Zapallal.

$\begin{array}{lll}\text { Fray } \mathrm{PE} \text {, et al. } & 2018 & \text { Revisión bibliográfica. } \\ & \text { Ecuador } & \text { Sistematizar el accionar de }\end{array}$ Licenciatura enfermería en la atención del parto culturalmente adecuado según el criterio de los diferentes autores.

Quiñonez FJ, 2018 Cuanti-cualitativo, transversal et al. $31 \quad$ Ecuador

Licenciatura

descriptivo. Evaluar los conocimientos y prácticas del cuidado materno infantil a niños de 0 a 3 años en el barrio 24 de mayo del cantón Esmeraldas.

Palomino 2018, SM, et al. ${ }^{32}$ Perú Licenciatura
Estudio cuantitativo, diseño no experimental descriptivo trasversal.

Conocer los factores asociados a su abandono es esencial para identificar los grupos vulnerables que requieren de intervención del enfermero (a).
35 documentos.

30 madres 0 cuidadores a cargo de niños/as.

madres tienen el debido conocimiento a qué edad darle o introducirle la alimentación a sus hijos, mientras que el $7 \%$ prefirió esperar a que sus hijos tuviera una edad en la que puedan digerir adecuadamente los alimentos

55 madres con niños menores de 6 meses de edad.

Existe asociación del abandono de la lactancia materna exclusiva con los factores demográficos y sus indicadores edad de la madre $(0,042)$, edad del padre (0.000), grado de instrucción del padre (0.008) y situación laboral de la madre (0.030); el factor social y apoyo familiar con su indicador niño menor de 6 meses con cólicos o gases recibe agüita de anís (0.040); el factor físico con su indicador enfermedad que le impida la lactancia materna (0.000) y el Factor rutina hospitalaria y apoyo profesional con el indicador tiempo separada de su hijo después del parto (0.004). 
Tabla 1. Continuación....

\begin{tabular}{lll}
\hline $\begin{array}{l}\text { Abanto SL, et } \\
\text { al. }{ }^{33} \\
\text { Licenciatura }\end{array}$ & $\begin{array}{l}\text { 2017, } \\
\text { Perú }\end{array}$ & $\begin{array}{l}\text { Estudio Cuantitativo, } \\
\text { descriptivo, transversa. }\end{array}$ \\
& $\begin{array}{l}\text { Objetivo: Conocer el nivel de } \\
\text { conocimientos que tienen las } \\
\text { madres sobre cuidados } \\
\text { domiciliarios al recién nacido } \\
\text { pretermino }\end{array}$
\end{tabular}

Colorado LD, 2017, et al. ${ }^{34} \quad$ Colombia

Licenciatura

$\begin{array}{lll} & \\ & & \\ & \\ \text { Pérez BR, et 2016, } \\ \text { al. } 36 & \text { Perú } \\ \text { Licenciatura } & \end{array}$

$\begin{array}{lll} & \\ & & \\ & \\ \text { Pérez BR, et 2016, } \\ \text { al. } 36 & \text { Perú } \\ \text { Licenciatura } & \end{array}$

$\begin{array}{lrr} & & \\ & & \\ & & \\ & & \\ \text { Pérez BR, et } & 2016, \\ \text { al. } & 36 & \text { Perú } \\ \text { Licenciatura } & \end{array}$

Niño CL, et 2017

Licenciatura

Perú

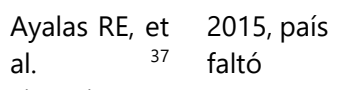

Licenciatura
Estudio cualitativo, diseño no experimental, transversal.

Caracterizar las concepciones sobre "humanización", "cuidado humanizado de la salud" y "formación ciudadana" en estudiantes y profesores del programa de Enfermería de la Universidad Católica de Manizales.

Estudio relacional, observacional, transversal y prospectivo.

Determinar la relación entre los conocimientos y las prácticas sobre el cuidado de los recién nacidos en madres adolescentes que acuden al Centro de Salud de Pillcomarca-Huánuco, 2016.

Estudio descriptivo simple.

Determinar el desempeño de las madres primíparas de 18 a 25 años en el cuidado del recién nacido en el servicio de neonatología del Hospital Regional Hermilio Valdizán Medrano de Huánuco en el 2016.

Estudio cuantitativo, descriptivo, comparativo transversal.

Comparar el cuidado que brindan las enfermeras en el servicio de cirugía del Hospital Víctor Lazarte con el cuidado que brindan las enfermeras del Hospital Belén de Trujillo en adultos postatectomizados.
178 estudiantes $\mathrm{y}$ 14 profesores del programa de enfermería.

50 madres de adolescentes.

75 madres primíparas de 18 a 25 años de edad.

98 adultos

(nd
En dimensión seguridad y protección el $75,3 \%$ de las madres tienen un nivel de conocimiento medio. En dimensión confort el $71,0 \%$ tienen un nivel de conocimiento medio. La dimensión alimentación el 60,2\% las madres tienen un nivel de conocimiento medio. En la dimensión Vínculo afectivo el $60,2 \%$ de las madres tienen un nivel de conocimiento medio. Se concluye por lo tanto que el $65,6 \%$ de las madres tienen un nivel de conocimiento medio y el $12,9 \%$ un nivel de conocimiento bajo. El mayor porcentaje de las madres evaluadas refirió un conocimiento medio sobre cuidados domiciliarios al recién nacido pretérmino

Después del análisis observado, muestran una prevalencia, en la categoría de Humanización, seguida de la Formación Ciudadana y por último la categoría de Cuidado Humanizado de la Salud, emergiendo la categoría Otros, en algunas respuestas.

$60 \%$ de madres adolescentes tuvieron conocimientos buenos y el $40 \%$ conocimientos deficientes sobre el cuidado del recién nacido; respecto a las prácticas, el $64 \%$ tuvieron prácticas adecuadas y el $36 \%$ prácticas inadecuadas; los conocimientos se relacionaron significativamente con las prácticas de cuidado de los recién nacidos.

El $65,3 \%$ madres primíparas tuvieron un buen nivel de desempeño en el cuidado del recién nacido, siendo estadísticamente significativo $(p=0,0008)$.

no hubo diferencia significativa en la subescala sanador compasivo, solo en la subescala sanador competente. 
Tabla 1. Continuación....

\begin{tabular}{|c|c|c|}
\hline $\begin{array}{l}\text { Mamani PJ. }{ }^{38} \\
\text { Licenciatura }\end{array}$ & 2015, Perú & $\begin{array}{l}\text { Estudio cuantitativo, descriptivo, } \\
\text { comparativo, transversal. } \\
\text { Analizar los conocimientos y } \\
\text { actitudes de madres y padres sobre } \\
\text { lactancia materna en el Hospital } \\
\text { Hipólito Unanue de Tacna } 2015 \text {. }\end{array}$ \\
\hline $\begin{array}{l}\text { Mansillas RR, } \\
\text { et al. }{ }^{39} \\
\text { Especialidad }\end{array}$ & 2019, Perú & $\begin{array}{l}\text { Estudio cuasi experimental. } \\
\text { Medir las necesidades del familiar } \\
\text { del paciente en UCl, aplicar sesiones } \\
\text { de cuidado según modelo de } \\
\text { Kristen Swanson y analizar el nivel } \\
\text { de satisfacción logrado. }\end{array}$ \\
\hline $\begin{array}{l}\text { Marín PG, et } \\
\text { al. } \\
\text { Especialidad }\end{array}$ & $\begin{array}{l}2018, \\
\text { Colombia } \\
\text { país faltó }\end{array}$ & $\begin{array}{l}\text { Marco lógico. } \\
\text { Diseñar una herramienta educativa } \\
\text { dirigida a los padres sobre cuidados } \\
\text { básicos de enfermería en el recién } \\
\text { nacido. }\end{array}$ \\
\hline
\end{tabular}

Mansillas RR, 2019, Perú

et al. ${ }^{39}$ nacido.

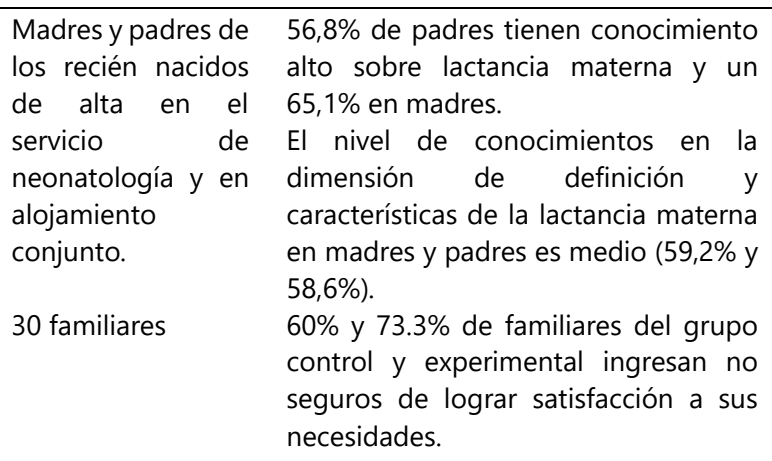

30 familiares

\begin{abstract}
$56,8 \%$ de padres tienen conocimiento alto sobre lactancia materna y un $65,1 \%$ en madres.

El nivel de conocimientos en la dimensión de definición y características de la lactancia materna en madres y padres es medio $(59,2 \%$ y $58,6 \%)$.

$60 \%$ y $73.3 \%$ de familiares del grupo control y experimental ingresan no seguros de lograr satisfacción a sus
\end{abstract} necesidades.

Estudio cuantitativo, cuasi experimental. Determinar la efectividad de la intervención educativa en el conocimiento del cuidador primario sobre los cuidados del paciente post operado del servicio de hospitalización de cardiopediatría en el Instituto Nacional de Salud del Niño San Borja de octubre - noviembre 2018.

Ponte VK, et 2017, Perú Estudio cuantitativo, descriptivo, al. 42 transversal. Determinar los efectos Especialidad

del masaje Shantala en el nivel de dolor en lactantes menores de dos años sometidos a ventilación mecánica.

$\begin{array}{ll}\text { Hooker ZL, et } & 2018, \\ \text { al. 43 } & \text { Colombia } \\ \text { Especialidad } & \text { país faltó }\end{array}$

Reategui AJ, 2018, Perú et al. ${ }^{40}$

Especialidad
Marco lógico.

Árbol de objetivos: Asegurar una buena alimentación del recién nacido con leche materna cuando la mamá no se encuentre en la hospitalización.

Estudio cuantitativo, no experimental.

Determinar la asociación entre la calidad del cuidado de enfermería y la estancia hospitalaria del neonato prematuro en la $\mathrm{UCI}$ neonatales del Hospital Regional de Loreto.
Matriz de marco lógico.

58 cuidadores primarios de pacientes pediátricos.

30 lactantes.

Revisión

bibliográfica.

15 enfermeras que laboran en la UCI neonatales.
Las enfermeras que laboran en turnos rotativos aplicaran la guía adaptada del masaje Shantala con validez de contenido a los lactantes que se encuentren con ventilación mecánica invasiva en modo PSV y CPAP, se logró aliviar el dolor favoreciendo su desarrollo neurológico y fomentando esta actividad de enfermería como una atención innovadora y humanizada,

Mediante la revisión bibliográfica y la orientación por el grupo de expertos, se realizó la guía de manejo y conservación de leche materna.

Protocolo, no hay resultados. 
Tabla 1. Continuación....

\begin{tabular}{|c|c|c|}
\hline $\begin{array}{l}\text { Flores HG, et } \\
\text { al. } \\
\text { Especialidad }\end{array}$ & $\begin{array}{l}2017, \\
\text { Colombia } \\
\text { país faltó }\end{array}$ & $\begin{array}{l}\text { Matriz del marco lógico. } \\
\text { Unificar la intervención "cuidado madre } \\
\text { canguro" para la atención de los niños } \\
\text { prematuros y/o de bajo peso } \\
\text { hospitalizados en una institución de } \\
\text { cuarto nivel. }\end{array}$ \\
\hline $\begin{array}{l}\text { Vargas BD, et } \\
\text { al. } \\
\text { Especialidad }\end{array}$ & $\begin{array}{l}2016, \\
\text { Colombia }\end{array}$ & $\begin{array}{l}\text { Revisión de literatura. } \\
\text { Elaborar una guía de cuidado interactiva } \\
\text { humanizada para la toma de gases } \\
\text { arteriales aplicando la TC para } \\
\text { estudiantes de pregrado y posgrado de } \\
\text { la facultad de enfermería de la } \\
\text { Universidad Nacional de Colombia, } \\
\text { generando una herramienta educativa } \\
\text { de simulación clínica que permita } \\
\text { ofrecerle al paciente un procedimiento } \\
\text { seguro y humanizado. }\end{array}$ \\
\hline $\begin{array}{l}\text { Arellano MA, } \\
\text { et al. } 47 \\
\text { Especialidad }\end{array}$ & $\begin{array}{l}\text { 2016, } \\
\text { Colombia }\end{array}$ & $\begin{array}{l}\text { Estudio descriptivo. } \\
\text { Establecer un plan de mejora para } \\
\text { intervenir las dimensiones "explica y } \\
\text { facilita" y "se anticipa" del instrumento } \\
\text { "CARE Q" a través de actividades que } \\
\text { inviten al personal enfermero a mejorar } \\
\text { la calidad del cuidado y establecer una } \\
\text { comunicación efectiva con los padres de } \\
\text { los neonatos hospitalizados en el área } \\
\text { de cuidados especiales neonatales de la } \\
\text { Clínica Universitaria Bolivariana, durante } \\
\text { el periodo comprendido entre febrero - } \\
\text { mayo de } 2016 \text {. }\end{array}$ \\
\hline $\begin{array}{l}\text { Reyes OA, et } \\
\text { al. } \\
\text { Especialidad }\end{array}$ & $\begin{array}{l}\text { 2016, } \\
\text { Colombia }\end{array}$ & $\begin{array}{l}\text { Metodología del marco lógico. } \\
\text { Diseñar, implementar y evaluar una } \\
\text { estrategia educativa que favorezca el } \\
\text { cuidado de enfermería a los padres con } \\
\text { hijos en situación de muerte inminente } \\
\text { en la UCl pediátrica del Hospital } \\
\text { Universitario de Santander. }\end{array}$ \\
\hline
\end{tabular}

Aguirre SE, et 2018, al. ${ }^{49}$ Magister Perú

Estudio cuantitativo, tipo de estudio aplicada, explicativa, experimental-

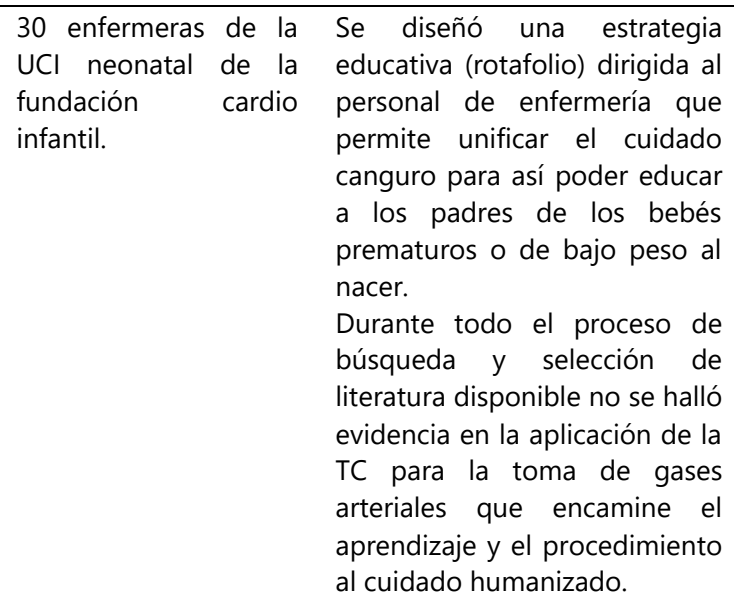

Padres (madre y/o padre) del neonato hospitalizado en el servicio de cuidados especiales, con más de 48 horas en el servicio $y$ aquellos que fueron dados de alta o trasladados a otro servicio.

Padres con hijos en situación de muerte inminente preexperimental.

Determinar la eficacia del programa: preparación de madres para mejorar el cuidado del recién nacido prematuro en casa, servicio de neonatología Hospital Loayza, Lima 2015.

30 madres de recién nacidos prematuros, entre octubre a noviembre del año 2015.

10 madres atendidas

Estudio pre- experimental.

$\begin{array}{ll}\text { Espinoza CM, } & 2018 \\ \text { et al. }{ }^{50} & \text { Perú }\end{array}$ Magister

Determinar la efectividad del Programa "Mamá canguro" sobre los conocimientos, prácticas y satisfacción con el cuidado del neonato, en madres de una clínica particular de Lima, 2018. desde diciembre del

2017 hasta marzo del 2018.
La dimensión "explica y facilita" del instrumento "CARE Q" tuvo un resultado satisfactorio del $90 \%$ el cual superó el $73,6 \%$. La dimensión "se anticipa" arrojó un resultado del $96.6 \%$, que superó el $71,9 \%$.

Al brindar un adecuado acompañamiento a los padres de niños en situación de muerte inminente se disminuyen los sentimientos negativos, favoreciendo la tranquilidad $y$ un entorno de mayor calidez en esos momentos difíciles.

Al comparar al grupo experimental en las dos condiciones (antes y después de la aplicación del programa) se evidencian diferencias altamente significativas $\mathrm{p}<$ 0.000). Los rangos negativos indican que el $100 \%$ de las madres mejoraron el cuidado del recién nacido prematuro.

El nivel de conocimientos, prácticas y satisfacción en las madres ingresadas en el programa mamá canguro, tuvo una efectividad del $100 \%$ 
Tabla 1. Continuación....

\begin{tabular}{|c|c|c|c|c|}
\hline $\begin{array}{l}\text { Humán } \mathrm{HD} \text {, } \\
\text { et al. } 51 \\
\text { Magister }\end{array}$ & $\begin{array}{l}\text { 2017, } \\
\text { Perú }\end{array}$ & $\begin{array}{l}\text { Estudio Cuasi experimental diseño } \\
\text { pre y post-test. } \\
\text { Evaluar el cuidado enfermero } \\
\text { basado en la TC en la mejora de la } \\
\text { aceptación de la práctica de la } \\
\text { lactancia materna de las madres } \\
\text { primigestas del hospital Augusto } \\
\text { Hernández Mendoza - EsSalud. } \\
\text { Ica } 2016 \text {. }\end{array}$ & 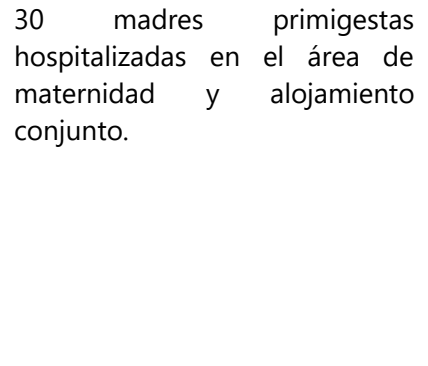 & $\begin{array}{l}63.3 \% \text { de madres presentaron } \\
\text { regular nivel de conocimiento } \\
\text { de la lactancia materna. En la } \\
\text { primera lista de chequeo sobre } \\
\text { la aceptación de la práctica de la } \\
\text { lactancia materna de las madres } \\
\text { primigestas existe } 80 \% \text { de } \\
\text { aceptación; mientras que en la } \\
\text { segunda lista existe } 70 \% \text { de } \\
\text { aceptación. }\end{array}$ \\
\hline $\begin{array}{l}\text { Diaz OM. }{ }^{52} \\
\text { Magister }\end{array}$ & $\begin{array}{l}\text { 2016, } \\
\text { Perú }\end{array}$ & $\begin{array}{l}\text { Estudio cuantitativo, descriptivo, } \\
\text { no experimental y correlacional. } \\
\text { Determinar la relación entre la } \\
\text { habilidad de cuidado y la carga } \\
\text { del cuidador primario informal } \\
\text { familiar (CPIF) de los pacientes } \\
\text { con enfermedad crónica } \\
\text { pertenecientes al programa } \\
\text { "Familiar Acompañante" del } \\
\text { Hospital Nivel II Vitarte - EsSalud, } \\
2015 \text {. }\end{array}$ & $\begin{array}{l}\text { Todos los familiares cuidadores } \\
\text { de los pacientes adultos mayores } \\
\text { de } 60 \text { años, atendidos en el } \\
\text { servicio de Hospitalización que } \\
\text { padecen de una enfermedad } \\
\text { crónica y que están registrados } \\
\text { en el programa familiar } \\
\text { acompañante durante los meses } \\
\text { de julio a octubre del año } 2015 \text {. }\end{array}$ & $\begin{array}{l}\text { No existe relación entre la } \\
\text { habilidad de cuidado y la carga } \\
\text { del cuidador } p=0.099) \text {. }\end{array}$ \\
\hline $\begin{array}{l}\text { Rojas EB, et } \\
\text { al. }{ }^{53} \\
\text { Doctorado }\end{array}$ & $\begin{array}{l}\text { 2017, } \\
\text { México }\end{array}$ & $\begin{array}{l}\text { Estudio mixto, método } \\
\text { hermenéutico. Objetivo: Describir } \\
\text { los cuidados e intervenciones de } \\
\text { enfermería enfocados en las } \\
\text { necesidades de salud de los } \\
\text { adultos mayores que asisten a una } \\
\text { casa de día en Toluca para diseñar } \\
\text { un modelo de cuidados e } \\
\text { intervenciones de enfermería } \\
\text { fundamentado en la TC. }\end{array}$ & $\begin{array}{l}177 \text { mujeres de } 65 \text { a } 84 \text { años de } \\
\text { edad de la casa de día. }\end{array}$ & $\begin{array}{l}\text { Se propone el Modelo de } \\
\text { Cuidado para el Adulto Mayor } \\
\text { sustentado en la TC la } \\
\text { importancia del estudio radica } \\
\text { en la obtención de un } \\
\text { instrumento validado para la } \\
\text { valoración del adulto mayor } \\
\text { sano, así como, la propuesta de } \\
\text { un modelo de cuidado basado } \\
\text { en la TC }\end{array}$ \\
\hline
\end{tabular}

Fuente: Elaboración propia

En cuanto a la aportación en el área clínica, surgió una escala del cuidado para identificar el cuidado que brindan las enfermeras ${ }^{(37)}$, se elaboró "la guía de cuidado interactiva de gasometría arterial basada en la TC" refiriendo que es una herramienta de fácil acceso y novedosa que permitirá al estudiante afianzar conocimientos y enfocarse en el trato al paciente ${ }^{(46)}$; contemplando los procesos básicos, ayuda a la identificación de los elementos del cuidado en la práctica (valoración, diagnostico resultados, intervenciones y evaluación) ${ }^{(14)}$, la TC permite al profesional de enfermería adoptar conductas modelo para las madres ya que, a través de los cinco procesos básicos del cuidado, se modifican progresivamente las conductas (38); surge el instrumento diseñado para medir el impacto del aborto involuntario $(12,19,18)$. 
En el área de docencia el aplicar la teoría permitió la elaboración de una "Guía de manejo para la extracción y conservación de la leche materna ${ }^{(43)}$. Al realizar intervenciones utilizando en su totalidad los procesos básicos se identificaron necesidades a nivel físico, familiar y social, que estarían afectando el desarrollo gestacional ${ }^{(55)}$; la garantía del cuidado mejora significativamente aplicando la TC ${ }^{(39)}$, se mejora la aceptación de la práctica de la lactancia materna de las madres primigestas ${ }^{(51)}$.

En el ámbito comunitario se sugiere incluir cuidados domiciliarios aplicando la TC por parte del profesional de enfermería, para que los pacientes no abandonen su tratamiento(29); se observó que la TC "se encuentra íntimamente relacionada con el cuidado que ejerce la enfermería en cuanto a la educación y la práctica, en su contexto clínico y colectivo, aborda al individuo y a su familia de forma holística" (40) incorporar la TC en investigaciones uniendo la docencia y la práctica hospitalaria, para detectar deficiencias del cuidado de enfermería que reciben los pacientes hospitalizados en los diferentes servicios de hospitalización y poder mejorarlo(25) ; la TC resulta apta para poder apoyar a las personas que viven la pérdida de un hijo(15). A nivel comunitario la TC dirigido a mujeres mayores se fundamenta en: el diálogo, esperanza, movimiento y orientación de salud centrado en el ejercicio de la autonomía ${ }^{(53)}$.

\section{Discusión}

En base al análisis de la evidencia disponible de la literatura científica sobre la aplicación de la TC de Kristen Swanson en las áreas del quehacer de enfermería, se ha podido demostrar su gran utilidad y aplicabilidad para fundamentar y proporcionar cuidado holístico. En el área asistencial, algunos autores demostraron resultados satisfactorios aplicando la TC ${ }^{(47)}$, en la práctica materna y el cuidado infantil la TC contribuyó al fortalecimiento de los fundamentos teóricos y filosóficos sustentando los cuidados de enfermería (14). En la docencia, es importante incorporar la TC en los programas de enfermería para fortalecer la curricula

(34). En el área de administración, algunos autores, plasmaron la teoría en su problemática sin embargo, ya no la volvieron a mencionar en sus intervenciones ${ }^{(48)}$; se dio origen a una guía de cuidado interactiva para la toma de gases arteriales en programas de educación ${ }^{(46)}$, en investigaciones de intervención donde 
evaluaron conocimientos para después reforzarlos fue de mucha ayuda la TC, su aplicación conlleva a una reflexión sobre los conocimientos y actitudes sobre la lactancia materna ${ }^{(38)}$, lo ideal es que se aplique la TC completa, está debe permear desde un inicio y a lo largo de la misma, hasta llegar a la propuesta, es decir, con sus cinco procesos básicos; conocimientos, estar con, hacer por, posibilitar y mantener las creencias (39), esto permite reflexionar sobre el cuidado proporcionado por enfermería. También existe evidencia de la efectividad del cuidado domiciliario en la adherencia al tratamiento en pacientes hipertensos ${ }^{(29)}$.

La TC surgió de la fenomenología, por lo tanto, ayuda a entender desde los significados que las personas atribuyen a los procesos que viven, así permitirá llevar con éxito las intervenciones planteadas, por lo que

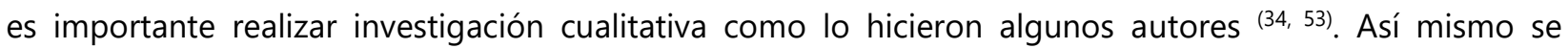
identificó que hubo modificación de la actitud hacia la donación de órganos en estudiantes ${ }^{(24)}$. Sin importar la experiencia que tenga en ese momento la enfermera o enfermero, el cuidado se brinda con la misma calidad y calidez.

\section{Conclusiones}

La presente investigación permitió sintetizar y analizar la literatura científica disponible, en idioma inglés y español, respecto a la aplicación de la TC en las cuatro áreas del quehacer de enfermería; la evidencia presentada puede ser de utilidad para investigadores y personal de enfermería interesado en implementar una teoría propia de la disciplina de enfermería a sus intervenciones, independientemente del área de desempeño.

Los profesionales de enfermería deben esforzarse por llevar las teorías de enfermería a la práctica para fortalecer la disciplina; y a la propia teoría de rango medio de Kristen Swanson; la práctica basada en teoría se retroalimenta y ayuda a comprender experiencias y fenómenos en las diferentes áreas cuando se brinda el cuidado, la teoría llevada a la práctica genera conocimiento empírico de la disciplina, provee conocimiento y fundamenta los cuidados proporcionados a las personas. 
Los hallazgos encontrados en la presente revisión de literatura contribuyen a enriquecer el cuerpo de conocimientos de enfermería, porque se encuentra íntimamente relacionada con el cuidado que ejerce enfermería al proporcionar cuidado a las personas, familias y comunidad de forma holística.

La TC se considera esencial para la valoración integral al momento de proporcionar cuidado, el adoptar los fundamentos y conceptos de la teoría son de gran utilidad a la hora de brindar acompañamiento cálido y humanizado a las personas por esto es relevante utilizarla ya que favorece la generación de un primer espacio de aprendizaje, resulta útil para tener un acompañamiento con el paciente y su familia, y ofrecerles estrategias de mejora desde la TC. Además, es importante que se realicen más investigaciones de intervención y evaluar dichas intervenciones, el educar y evaluar lo aprendido por la población genera respuestas propias de un grupo que pueden ser generalizados; en la docencia se requieren trabajar más en esta área e incluir la teoría en planes de estudio.

\section{Conflicto de intereses}

Los autores declaran que no existe conflicto de interés.

\section{Financiamiento}

Sin financiamiento

\section{Referencias bibliográficas}

1. Marriner A, Raile M. Modelos y Teorías en Enfermería. Harcourt Ged. Madrid. España: 2007.

2. Villalobos DM. Enfermería desarrollo teórico e investigativo. Universidad Nacional de Colombia Santafé de Bogotá, D. C. Proyecto INNOVAR. 2001

3. Carvajal VA. Teoría y modelos: Formas de representación de la realidad. Comunicación [Internet]. 2002 [citado 19 may 2020];12(001):1-14. Disponible en: https://www.redalyc.org/pdf/166/16612103.pdf

4. Kérouac S, Pepin J, Ducharme F, Duquette A, Major F. El pensamiento enfermero. Barcelona, España. Elseiver Masson. 2002.

5. Moreno FM, Muñoz de Rodríguez L, Alemán CP, Avila ML, Badillo RK, Barajas L. et al. De la teoría de enfermería a la práctica. Bogota: Universidad de La Sabana [Internet]. 2016 [citado 13 abr 2020]. Disponible en: https://publicaciones.unisabana.edu.co/publicaciones/publicaciones/enfermeria-y-rehabilitacion/de-lateoria-de-enfermeria-a-la-practica/ 
6. Fawcett J. Contemporary nursing knowledge: Analysis and evaluation of nursing models and theories. 2 ed. Philadelphia: FA Davis Company; 2005.

7. Durán de Villalobos M. Teoría de enfermería: ¿un camino de herradura?. Aquich [Internet]. 2007 [citado 03 abr 2020];7(2):161-173. Disponible en: http://www.scielo.org.co/scielo.php?script=sci arttext\&pid=S165759972007000200005\&lng=es.

8. Rodríguez C V, Valenzuela SS. Teoría de los cuidados de Swanson y sus fundamentos, una teoría de mediano rango para la enfermería profesional en Chile. Enferm glob [Internet]. 2012 [citado 05 mar 2020];11(28):316-322. Disponible en: http://scielo.isciii.es/scielo.php?script=sci arttext\&pid=S169561412012000400016\&lng $=$ es

9. Arce N, Carmona K. Percepción del enfermero(a) acerca del cuidado que requieren las personas en el servicio de recuperación, desde las áreas del quehacer de enfermería [Tesis de maestría]. Costa Rica: Universidad de Costa Rica [Internet]. 2019 [citado 19 may 2020]. Disponible en: http://repositorio.ucr.ac.cr/handle/10669/79224

10. Cooper $\mathrm{H}$. Research synthesis and meta-analysis a step by step approach. Applied social research methods series. 4a ed. Thousand, Oaks: Sage Publications, Inc.; 2010.

11. Wei $\mathrm{H}$, Roscign $\mathrm{Cl}$, Swanson K, Black BP, Hudson BD, Hanson Ch. Parents' experiences of having a child undergoing congenital heart surgery: An emotional rollercoaster from shocking to blessing. Heart \& Lung: The Journal of Cardiopulmonary and Acute Care [Internet]. 2016 [citado 24 jun 2019];45(2):154-160. Disponible en: https://doi.org/10.1016/j.hrtlng.2015.12.007

12. Wei Holly, Roscign Cl, Kristen M. Swanson. Healthcare providers' caring: Nothing is too small for parents and children hospitalized for heart surgery. Heart \& Lung: The Journal of Cardiopulmonary and Acute Care [Internet]. $2017 \quad$ [citado 19 may 2019];46(3):166-171. Disponible en: https://doi.org/10.1016/j.hrtlng.2017.01.007

13. Aguilera J, García M, Cáceda C. La familia como determinante social del intento de suicidio en adultos jóvenes: una mirada desde la complejidad. Cien Tecn [Internet]. 2016 [citado 19 may 2019];12(1):161-175. Disponible en: http://revistas.unitru.edu.pe/index.php/PGM/article/view/1368/

14. Costa OT, Lopes da SA, Da Silva OJ, Tenorio PE, Soares FM. A assistência de enfermagem obstétrica à luz da teoría dos cuidados de Kristen Swanson. Enferm Foco [Internet]. 2018 [citado 19 may 2019];9(2):03-062. Disponible en: http://revista.cofen.gov.br/index.php/enfermagem/article/view/1138/

15. Hernández LS. Vivencia del cuidado de enfermería en un proceso de duelo. Cul Cui [Internet]. 2019 [citado 10 ene 2020];23(54):50-66 Disponible en: http://hdl.handle.net/10045/96328

16. Rojas EJ, García HL, Cárdenas BL, Vázquez GS, Kempfer SS. Adaptación del modelo de Kristen Swanson para el cuidado de enfermería en adultas mayores. Contexto Enferm, [Internet]. 2018 [citado 18 may 2019];27(4):e0660017. Disponible: http://dx.doi.org/10.1590/0104-07072018000660017

17. Higgins JPT, Green S. (edit.). Cochrane Handbook for Systematic Reviews of Interventions. UK: The Cochrane Collaboration, [Internet]. 2011 [citado 03 ago 2016]. Disponible en: http://bit.ly/2jvMiad

18. Huffman CS, Schwartz TA, Swanson KM. Couples and miscarriage: The influence of gender and reproductive factors on the impact of miscarriage. Women's Health Issues [Internet]. 2015 [citado 18 may 2019];25(5):570-578. DOI: https://doi.org/10.1016/j.whi.2015.04.005

19. Wei $\mathrm{H}$, Roscign $\mathrm{Cl}$, Hanson $\mathrm{CC}$, Swanson K. Families of children with congenital heart disease: A literature review. Heart \& Lung: The Journal of Cardiopulmonary and Acute Care [Internet]. 2015 [citado 24 junio 2019];44(6):494-511. DOI: https://doi.org/10.1016/j.hrtlng.2015.08.005 
20. Bogulski J, Steven G, Doug B, Bugner R, Clark L, Farrell L, Swanson K, David R. The Run Smart training program: effect on oxygen consumption and lower extremity biomechanics during running. Journal of Exercise Rehabilitation [Internet]. 2017 [citado 18 may 2019];13(4):446-453. Disponible en: https://doi.org/10.12965/jer.1734994.497

21. Jansson C, Volgsten H, Huffman C, Skoog SA, Swanson K. Validation of the revised impact of miscarriage scale for swedish conditions and comparison between swedish and american couples experiences after miscarriage, The European Journal of Contraception \& Reproductive Health Care [Internet]. 2017 [citado 24 jun 2019]; 22(6):412-417. DOI: https://doi.org/10.1080/13625187.2017.1409346

22. Jinbing B, Swanson K, Felicity W.K Harper. Longitudinal analysis of parent communication behaviors and child distress during cancer port start procedures. Pain Management Nursing [Internet]. 2018 [citado 22 jun 2019];19(5):487-496. Disponible en: https://doi.org/10.1016/j.pmn.2018.01.002

23. Castaño C. Villegas A, Díaz V, González C, Noreña A, Ocampo CC, et al. Evaluación del proceso educativo hacia la madre sobre los cuidados en el puerperio y del recién nacido en ASSBASALUD E.S.E Manizales (Colombia). Arch Med [Internet]. 2018 [citado 10 ene 2020];18(2):352-63. Disponible en: https://doi.org/10.30554/archmed.18.2.2734.2018

24. Aquino AY, Cajaña BK. Aplicación de la teoría de Kristen Swanson para el cambio de actitud hacia la donación de órganos. [Tesis de pregrado]. Perú: Universidad Nacional de San Agustín de Arequipa [Internet]. 2018 [citado 18 may 2019]. Disponible en: http://repositorio.unsa.edu.pe/bitstream/handle/UNSA/5135/ENaqapy.pdf?sequence=1\&isAllowed=y

25. Mogollon AL, Guzmán TS. Percepción del cuidado enfermero en pacientes hospitalizados en el servicio de cirugía: según la teoría de Kristen Swanson [Tesis de pregrado]. Chiclayo: Universidad Católica Santo Toribio de Mogrovejo [Internet]. 2019 [citado 15 nov 2019]. Disponible en: http://hdl.handle.net/20.500.12423/1977

26. Romero AR, Torres LM, Huertas AF. Conocimientos y prácticas sobre lactancia materna y estado nutricional en lactantes menores [Tesis de pregrado]. Perú: Universidad Nacional de Trujillo [Internet]. 2019 [citado 19 may 2019]. Disponible en: http://dspace.unitru.edu.pe/handle/UNITRU/11636

27. Ding CS, Sandoval GE, Vélez DC. Practicas alimentarias de cuidadores con menores de 5 años de la comunidad Wayuu de Mayabangloma [Tesis de pregrado]. Fonseca: La Guijara. Corporación Universitaria Adventista [Internet]. 2018 [citado 19 may 2019]. Disponible en: http://repository.unac.edu.co/bitstream/handle/11254/101/Trabajo\%20de\%20grado.pdf?sequence=1\&isAl lowed =y

28. Bajaña $A Z$, Micolta RD, Ramírez PT. Intervención de enfermería en la seguridad del neonato en alojamiento conjunto del Hospital Pablo Arturo Suarez [Tesis de pregrado]. Ecuador: Universidad de Guayaquil [Internet]. 2018 [citado 10 oct 2019]. Disponible en: http://repositorio.ug.edu.ec/handle/redug/34650

29. Chinguel YK, Peredo RB. Efectividad del cuidado domiciliario de enfermería en la adherencia al tratamiento según la teoría de Swanson en pacientes con hipertensión arterial en un centro de salud de puente piedra [Tesis de pregrado]. Perú: Universidad Peruana Cayetano Heredia [Internet]. 2018. [citado 05 dic 2019]. Disponible en: http://repositorio.upch.edu.pe/bitstream/handle/upch/3809/Efectividad ChinguelLizana Karem.pdf?seque $\underline{\text { nce }=1 \text { \&isAllowed }=y}$ 
30. Fray PE, Rivera HG. Accionar de enfermería en la atención del parto culturalmente adecuado [Tesis de pregrado]. Ecuador: Universidad Nacional de Chimborazo [Internet]. 2018 [citado 05 dic 2019]. Disponible en: http://dspace.unach.edu.ec/handle/51000/5051

31. Quiñonez FJ, Acosta SM. Evaluación del conocimiento y práctica del cuidado materno infantil a niños de 0 a 3 años en el barrio 24 de mayo del cantón Esmeraldas [Tesis de pregrado]. Ecuador: Esmeraldas Pontificia Universidad Católica del Ecuador [Internet]. 2018 [citado 18 may 2019]. Disponible en: https://repositorio.pucese.edu.ec/handle/123456789/1611

32. Palomino SM, Estrada ZS, Gálvez DN. Factores asociados al abandono de la lactancia materna exclusiva, centro de salud San Antonio [Tesis de pregrado]. Perú: Universidad Señor de Sipán [Internet]. 2018. [citado 18 may 2019].

Disponible

en:

http://repositorio.uss.edu.pe/bitstream/handle/uss/5027/Palomino\%20Santa\%20Cruz\%20\%26\%20Estrada $\% 20$ Sambrano.pdf? sequence $=1$ \&isAllowed $=y$

33. Abanto SL, Vinces LM, Gálvez DN. Conocimiento de las madres sobre los cuidados domiciliarios del recién nacido pre término en Lambayeque - Perú 2016. Gac Med Bol [Internet]. 2017 [citado 10 ene 2020];40(2):27-30. Disponible en: http://www.scielo.org.bo/scielo.php?script=sci arttext\&pid=S101229662017000200006\&lng=es

34. Colorado LD, Gil EX, Sánchez DM, Torres AD, Ruiz TM, Orozco DM. Concepción sobre la humanización del cuidado de la salud: en estudiantes y profesores del programa de enfermería de la universidad católica de Manizales [Tesis de pregrado]. Colombia: Universidad Católica de Manizales [Internet]. 2017 [citado 18 may 2019]. Disponible en: http://repositorio.ucm.edu.co:8080/jspui/handle/10839/1715

35. Niño $\mathrm{CL}$, Palma LD. Conocimientos y prácticas sobre el cuidado de los recién nacidos en madres adolescentes que acuden al Centro de Salud. [Tesis de pregrado]. Pillco Marca: Universidad de Huánunco [Internet]. 2017 [citado 18 may 2019]. Disponible en: http://repositorio.udh.edu.pe/123456789/626

36. Pérez BR, Carnero TA. Desempeño de primíparas de 18 a 25 años ante el cuidado del recién nacido en el servicio de neonatología del Hospital Regional Hermilio Valdizán Medrano [Tesis de pregrado]. Peru: Universidad de Huánuco [Internet]. 2016 [citado 17 may 2019]. Disponible en: http://repositorio.udh.edu.pe/123456789/132

37. Ayalas RE, Giles CK, Mendo ZT. Cuidado de enfermería en adultos postatectomizados en hospitales de Trujillo [Tesis de pregrado]. Perú: Universidad Nacional de Trujillo [Internet]. 2015 [citado 17 may 2019]. Disponible en: http://dspace.unitru.edu.pe/handle/UNITRU/5805

38. Mamani PJ. Conocimiento y actitudes de madres y padres sobre sobre lactancia materna en el Hospital Hipólito Unanue de Tacna [Tesis de pregrado]. Grohmann-Tacna: Universidad Nacional Jorge Basadre [Internet]. 2015 [citado 19 may 2019]. Disponible en: http://repositorio.unjbg.edu.pe/handle/UNJBG/2169 39. Mansillas RR, Velásquez RS. Aplicación de la teoría de los cuidados de Kristen Swanson en la satisfacción de necesidades en familiares de pacientes críticos. Unidad de cuidados intensivos Hospital Regional Honorio Delgado [Tesis de especialidad]. Perú: Universidad Nacional de San Agustín de Arequipa [Internet]. 2019 [citado 05 dic 2019]. Disponible en: http://repositorio.unsa.edu.pe/handle/UNSA/10511

40. Marín PG, Ramírez AG, Carranza GN. Herramienta educativa sobre cuidados básicos de enfermería en el recién nacido dirigida a padres de la unidad de cuidado intensivo e intermedio neonatal en un Hospital de IV nivel [Tesis de especialidad]. Bogotá: Universidad de la Sabana [Internet]. 2018 [citado 05 dic 2019]. Disponible en: https://intellectum.unisabana.edu.co/handle/10818/35440 
41. Guzmán OY, Soto PJ, Albino LM, Salazar AY. Efectividad de la intervención educativa en el conocimiento del cuidador primario sobre los cuidados del paciente post operado del servicio de Hospitalización de cardiopediatria. [Tesis de especialidad]. Perú: Universidad Peruana Cayetano Heredia [Internet]. 2018 [citado 18 may 2019]. Disponible en: http://repositorio.upch.edu.pe/handle/upch/3903

42. Ponte VK, Sagástegui MC, Vais BR. Efectos del masaje shantala en el dolor de los lactantes menores de dos años con ventilación mecánica en la unidad de cuidados intensivos del INSN [Tesis de especialidad]. Perú: Universidad peruana Cayetano Heredia [Internet]. 2017 [citado 10 ene 2020] Disponible en: http://repositorio.upch.edu.pe/bitstream/handle/upch/958/Efectos PonteVillanueva Kelly.pdf?sequence=3 \&isAllowed $=\mathrm{y}$

43. Hooker ZL, Quintero HY, Gutiérrez AM. Manejo para la extracción y conservación de la leche materna [Tesis de especialidad]. Cuba: Universidad de la Sabana [Internet]. 2018 [citado 5 may 2019]. Disponible en: http://hdl.handle.net/10818/33700

44. Reategui AJ, Ibérico AS, Tangoa AS, Sevillano BZ. Calidad del cuidado de enfermería y estancia hospitalaria del neonato prematuro en la unidad de cuidados intensivos neonatales del Hospital Regional [Tesis de especialidad]. Loreto: Universidad Peruana Unión [Internet]. 2018 [citado 19 may 2019]. Disponible en:

https://repositorio.upeu.edu.pe/bitstream/handle/UPEU/1097/Angelica Trabajo Investigación 2018.pdf?se quence $=5$ \&isAllowed $=y$

45. Flórez HG, Rivera FC, Ruiz de Cárdenas C. Estrategia educativa madre canguro, para el cuidado de enfermería de los niños prematuros o de bajo peso hospitalizados en la UCI neonatal de una institución de cuarto nivel. [Tesis de especialidad]. Cundinamarca: Universidad de la Sabana [Internet]. 2017 [citado 18 may 2019]. Disponible en: https://intellectum.unisabana.edu.co/bitstream/handle/10818/34828/Trabajo\%20de\%20grado\%20Ginna\% 20Florez\%20y\%20Cristina\%20Rivera.pdf?sequence=1\&isAllowed =y

46. Vargas BD, Parra DE. Guía de cuidado interactiva humanizada para la toma de gases arteriales, basados en la Teoría de Swanson, para estudiantes de la Facultad de Enfermería de la Universidad Nacional de Colombia [Tesis de especialidad]. Colombia: Universidad Nacional de Colombia [Internet]. 2016 [citado 05 dic 2019]. Disponible en: https://repositorio.unal.edu.co/handle/unal/58928

47. Arellano MA, Lara VJ, Jaramillo SL. Unidad de cuidados especiales neonatales: Un cuidado humanizado [Tesis de especialidad]. Medellín: Universidad Pontificia Bolivariana [Internet]. 2016 [citado 18 may 2019]. Disponible en: https://intellectum.unisabana.edu.co/handle/10818/25594

48. Reyes OA, Silva PJ, Abaunza de GM. Estrategia educativa que favorezca el cuidado de enfermería a los padres con hijos en situación de muerte inminente en la $\mathrm{UCl}$ pediátrica del Hospital Universitario de Santander. ¡Ayúdame a morir con dignidad! [Tesis de especialidad]. Bogotá: Universidad de la Sabana [Internet]. 2016 [citado 18 may 2019]. Disponible en: http://hdl.handle.net/10818/29741

49. Aguirre SE, Córdova SD. Eficacia del programa: preparación de madres para mejorar el cuidado del recién nacido prematuro en casa [Tesis de maestría]. Perú: Universidad César Vallejo [Internet]. 2018 [citado 19 may 2019]. Disponible en: https://repositorio.upeu.edu.pe/handle/UPEU/387

50. Espinoza CM, Bernui CM. Efectividad del programa "mamá canguro" en los conocimientos, prácticas y satisfacción sobre el cuidado del neonato, en madres de una clínica particular de lima [Tesis de maestría]. Lima: Universidad Peruana Unión [Internet]. 2018 [citado 19 may 2019]. Disponible en: http://repositorio.upeu.edu.pe/handle/UPEU/387 
51. Huamán HD, Velázquez RS. Cuidado enfermero durante la lactancia materna basada en la teoría de Kristen Swanson en madres primigestas del Hospital Augusto Hernández Mendoza - ESSALUD ICA [Tesis de Maestría]. Perú: Universidad Nacional de San Agustín de Arequipa [Internet]. 2016 [citado 06 dic 2019]. Disponible en: http://repositorio.unsa.edu.pe/handle/UNSA/6873

52. Diaz OM. Habilidad de cuidado y carga del cuidador primario informal familiar de los pacientes con enfermedad crónica del "Programa Familiar Acompañante" del hospital Nivel II Vitarte - EsSalud [Tesis de maestría]. Perú: Universidad Peruana [Internet]. 2016 [citado 06 dic 2019]. Disponible en: http://11 habilidad del cuidado.pdf

53. Rojas EB, García HM, Cárdenas BL, Vázquez GL. Cuidados e intervenciones de enfermería enfocados en las necesidades de salud del adulto mayor, bajo la Teoría de Kristen M. Swanson [Tesis doctoral]. Estado de México: Universidad Autónoma del Estado de México [Internet]. 2017 [citado 18 may 2019]. Disponible en: http://hdl.handle.net/20.500.11799/99139

54. Carreño M, Barreto O, Duran P, Támara O, Romero E. Habilidad de cuidado y sobrecarga en cuidadores familiares de personas con enfermedad crónica. Rev Cubana Enferm [Internet]. 2016 [citado 09 ene 2020];32(3): Disponible en: http://www.revenfermeria.sld.cu/index.php/enf/article/view/951

55. Jojoa G. El valor del cuidado de enfermería en un proyecto de vida. (ed) UNIMAR. 75-78 [Internet]. s/f [citado 18 2019]. Day en: http://editorial.umariana.edu.co/revistas/index.php/libroseditorialunimar/login

Cómo citar este artículo: Beristain-García I, Álvarez-Aguirre A, Huerta-Baltazar M,Casique-Casique L. Teoría de los cuidados de Kristen Swanson: revisión de literatura. SANUS [Internet]. 2022 [citado dd mm aa];7:e212. Disponible en: DOI/URL 